ARTíCULOS DE INVESTIGACIÓN

\title{
Experiencia de evaluación continua en cursos del ciclo inicial en la carrera de Derecho de la Universidad Católica de Temuco
}

\author{
Experiência de avaliação continuada em cursos do ciclo inicial do programa de \\ graduação da Universidad Católica de Temuco
}

An experience of continuous assessment in courses of the initial cycle of the undergraduatre Law Program at Universidad Católica de Temuco

\author{
Alejandra Cid Droppelmann \\ Universidad Católica de Temuco, Chile \\ Beatriz Moya Figueroa \\ Universidad Católica de Temuco, Chile \\ Patricia Toledo Zúñiga \\ Universidad Austral de Chile \\ Felipe Quintano Méndez \\ Universidad Católica de Temuco, Chile
}

RESUMEN El contexto actual en Educación Superior presenta diferencias que han incentivado a las instituciones a modificar sus formas de enseñanza, centrándolas en el estudiante. Si bien la formación de abogados no se encuentra ajena a estas transformaciones, sus modos de enseñanza aún están vinculadas prioritariamente hacia un enfoque tradicional, lo que se evidencia en sus mecanismos de evaluación. Ya que este es un elemento crítico, se realizó un pilotaje de evaluación continua en dos cursos de primer año de la carrera de Derecho de la Universidad Católica de Temuco y el presente estudio tiene por objeto evaluar su implementación. Para lograrlo, se analizaron las evaluaciones de los cursos con y sin la innovación por medio de una estadística descriptiva y otra correlacional. Además, se aplicaron dos cuestionarios con preguntas abiertas, uno al inicio y otro al final del semestre, enfocados en identificar la percepción de los estudiantes 
hacia la innovación. Los resultados denotan un mayor nivel de aprobación de los cursos con evaluación continua, con presencia de correlaciones entre evaluaciones que apuntan a habilidades de alto orden y al promedio final del curso. Finalmente, se identifica una percepción positiva de los estudiantes hacia la innovación.

PALABRAS CLAVE Evaluación continua, enseñanza del derecho, formación inicial de abogados.

RESUMO O contexto atual do ensino superior apresenta mudanças que encorajaram as instituições a modificarem os seus métodos de ensino, tornando-os mais centrados nos estudantes. A despeito de a formação dos juristas não estar alienada a estas transformações, os métodos de ensino ainda são essencialmente ligados à abordagem tradicional, o que evidencia-se especialmente nos métodos de avaliação. Sendo um elemento crítico, uma tentativa de avaliação continuada foi levada a cabo em duas turmas de primeiro ano de uma faculdade de Direito da UC Temuco. O presente estudo objetiva avaliar esta implementação. As avaliações dos cursos com e sem inovações foram realizadas por meio de estatísticas descritivas e correlacionais. Adicionalmente, dois questionários foram aplicados com questões abertas, um no início e outro no final do semestre, ambos focando em identificar a percepção dos estudantes com relação à inovação. Os resultados demonstraram um maior nível de aprovação em cursos com avaliação continuada, com a presença de correlações entre avaliações que apontam para maiores habilidades e a avaliação final do curso. Por fim, identificou-se uma percepção positiva dos estudantes diante da inovação.

PALAVRAS-CHAVE Avaliação continuada, Ensino do Direito, formação inicial dos juristas.

ABSTRACT The current context in Higher Education presents changes that have encouraged institutions to modify their teaching methods, making them more student-centered. Although the training of lawyers is not alien to these transformations, their teaching methods are still primarily linked to the traditional approach, which is especially evident in their evaluation mechanisms. Since this is a critical element, a continuous assessment piloting was carried out in two first-year courses of undergraduate Law program at Universidad Católica de Temuco. The present study aims to evaluate this implementation. The evaluations of the courses with and without innovations were analyzed through descriptive and correlational statistics. In addition, two questionnaires were applied with open questions, one at the beginning and the other at the end of the semester, focused on identifying the students' perception towards innovation. Results show a higher level of approval in courses with continuous assessment, with the presence of correlations between evaluations that point to higher order skills and the final average of the course. Finally, a positive perception of students towards innovation is identified.

KEYWORDS Continuous assessment, teaching of Law, initial education of lawyers. 


\section{Introducción}

En la actualidad, la formación de abogados interactúa con un contexto de cambios en la educación superior. Este marco no solo invita a las instituciones a modificar la concepción tradicional de lo que se entiende por enseñanza (Wiggins y McTighe, 2005), ${ }^{1}$ sino que también aquellas nociones que subyacen a la evaluación. La incorporación progresiva de un enfoque de enseñanza centrado en el estudiante ha sido la respuesta a los procesos de diversificación académica de los mismos (Fry, Ketteridge y Marshall, 2015). ${ }^{2}$

La enseñanza centrada en el estudiante se define como una mentalidad y una cultura dentro de las instituciones de educación superior, y un enfoque hacia el aprendizaje que es ampliamente relacionado y apoyado por teorías constructivistas (Hunt y Chalmers, 2013). De acuerdo con Brown (2011) en una revisión del trabajo de Weimer (2013), se establecen cinco aspectos que diferencian este enfoque del de carácter tradicional: el equilibrio del poder dentro del aula, la función del contenido del curso, el rol de docente y el rol del estudiante, la responsabilidad del aprendizaje y el propósito de la evaluación.

Respecto a lo anterior, el docente se presenta como un facilitador que apoya a un estudiante a cargo de la construcción de su propio aprendizaje, donde el contenido es utilizado como medio para desarrollar desempeños y no es un fin en sí mismo. Así, la evaluación no es sinónimo de calificación, sino que amplía su alcance como un elemento central que orienta el proceso de aprendizaje del estudiante (Hunt y Chalmers, 2013).

Para desarrollar este enfoque de enseñanza centrado en el estudiante, Nuckles (2000: 5) afirma que se deben considerar sus diferencias en los planos de habilidad intelectual, estilos y métodos de procesamiento cognitivo, personalidad, maneras de crear significado, características físicas, socialización, características de desarrollo y logros educacionales (Nuckles, 2000).

Más allá de tratarse de una lista exhaustiva de diferencias, las generalizaciones sobre los estudiantes son útiles para guiar la comprensión de los participantes de un programa o curso.

No obstante, en este escenario la enseñanza del derecho ha mantenido un modelo propio de formación, que tiende a un enfoque tradicional y presenta características que lo diferencian de otras disciplinas. Lo anterior se explica desde el concepto de

1. Como señalan Wiggins y McTighe, desde una perspectiva tradicional el concepto de enseñanza se entiende como aquella que se centra en la transmisión de la información. El foco está en la cobertura de los contenidos más que en la profundidad de los aprendizajes.

2. La diversidad se asocia a diferencias de tipo cultural, socioeconómico y académico. Esta noción es expandida por Heather Fry, Steve Ketteridge y Stephanie Marshall en el libro A Handbook for Teaching in Higher Education, publicado en 2015. 
pedagogías características (Shulman, 2005), que es definido como las formas de instrucción que se asocian de manera natural al momento de pensar en la preparación de los estudiantes de una profesión, revelando además las personalidades, disposiciones y culturas de las áreas de estudio (Shulman, 2005).

En la enseñanza del derecho, se evidencia una estructura superficial donde la formación del pensamiento, desempeño y actuación de los futuros abogados es llevada a cabo por un docente que interactúa sobre la base de preguntas y respuestas, escribiendo muy ocasionalmente en la pizarra y generando procesos de comunicación uno a uno, donde existe muy poca interacción entre los estudiantes en las actividades de aula (Shulman, 2005).

Por otra parte, Donald (2009) plantea que el derecho es la pedagogía de lo incierto, de la tensión, de la negociación, de la interpretación y el riesgo, y que está al servicio del desarrollo de habilidades de alto orden. Las habilidades de alto orden son completas e involucran acciones como el análisis y la síntesis, es decir, son reflexivas, sensibles al contexto y requieren de un automonitoreo, por tanto, se diferencian de otras que se llevan a cabo de manera mecánica (Halpern, 2007).

En el contexto anglosajón existen visiones críticas hacia el modo particular de la enseñanza del derecho que se centran, por ejemplo, en sus propósitos y escasa variabilidad. En relación con lo anterior, Shulman (2005) plantea que, si bien la educación legal favorece una formación que lleva a sus estudiantes a pensar como un abogado, adolece de una falta de preocupación hacia el desarrollo de desempeños. Además, Hunt y Chalmers (2013) resaltan la necesidad de que las escuelas de derecho desarrollen un enfoque más pluralista, que integre discusiones en las cátedras y que combine el método de casos con otras metodologías activas, como el seminario socrático. En relación con lo anterior, Gabilondo (2017) sostiene que el futuro de dicho método depende de la capacidad de los docentes del área para destacar su rol en la preparación de los abogados.

Por otra parte, en el contexto hispanoamericano los análisis se concentran en el carácter tradicional de la formación. En España,

Pérez Lledó identifica varias características del modelo de enseñanza jurídica como que: a) está basado en la transmisión y memorización de una gran cantidad de información acerca del contenido de normas (y de instituciones y conceptos jurídicos); b) se privilegia la lección magistral para la transmisión y recepción pasiva de información; c) se fomenta una educación formalista; d) es poco práctica (Ramírez Mejía, 2016: 35).

En nuestro país, en un artículo del año 2006, Alejandro Guzmán llamaba la atención sobre el carácter altamente conservador de la enseñanza del derecho en Chile. En casi 200 años, tanto el currículum de las escuelas de derecho como la metodología de enseñanza apenas habían experimentado cambios significativos (Benfeld, 2017). Del 
mismo modo, Gómez y Rubio (2017: 41) expresan que, en la enseñanza del derecho, este último adopta la característica de un sistema de normas que automatizan quién las dijo, por qué y para qué. En términos de paradigmas educativos, lo anterior ha desembocado en prácticas docentes instruccionales, en las que el aprender y el hacer aparecen como dos dimensiones lejanas y que solo se encuentran en momentos episódicos.

La forma en que se enseña el derecho, desde una perspectiva general, asume a la evaluación como un mecanismo de medición de logros y no como una herramienta que favorezca la construcción de nuevos aprendizajes (Coloma Correa, 2005). Las formas de evaluación a las que se recurre mayoritariamente son pruebas escritas y exámenes orales, en las que se privilegia la producción de contenido por parte de los estudiantes para efectos de generar una calificación (Coloma Correa, 2005).

Podemos señalar que el modelo de educación jurídica aparentemente está basado en aspectos formalistas y se propende a una labor más individualista de los estudiantes (Ramírez Mejía, 2016). Ahora bien, los instrumentos de evaluación que pretenden medir los aprendizajes bajo este modelo tienden a reproducir las características señaladas anteriormente (Ramírez Mejía, 2016).

Es importante destacar que, si bien se han implementado nuevas metodologías de enseñanza del derecho en Chile, nos encontramos con una dificultad, ya que no se ha variado en las formas de evaluar, especialmente en las instancias más importantes de la carrera, conservando los exámenes tradicionales e incluso manteniéndose el examen de grado en los mismos términos, es decir, considerando básicamente el viejo sistema memorístico/repetitivo (Pérez Villar, 2015).

Desde un marco más amplio, una clasificación que permite entender dos grandes categorías de la evaluación es ofrecida por Fink (2003). Desde esta mirada, la evaluación puede ser de medición o educativa. Una evaluación de medición se vincula a procedimientos de retroalimentación y evaluación que ocurren solo a medio término y al final de un curso. En este caso, el propósito es revisar el aprendizaje que los estudiantes han alcanzado con la finalidad de obtener una calificación.

La evaluación educativa contrasta con la propuesta anterior porque busca ayudar a los estudiantes a aprender mejor. En este marco, la evaluación es comprendida desde cuatro componentes principales: la evaluación prospectiva, la presencia de criterios y estándares, la presencia de autoevaluaciones y la retroalimentación frecuente, inmediata y discernidora (Fink, 2003).

De acuerdo con Biggs y Tang (2011), la evaluación permite generar cambios en los procesos de formación, ya que tiene un efecto en cómo los estudiantes comprenden y, a su vez, reaccionan ante los procesos de aprendizaje. La evaluación es lo primero que los estudiantes perciben en un proceso de formación, por tanto, orienta los énfasis de un curso (De Miguel y Apodaca, 2009; Brown, 2015; Padilla y Gil, 2008; Carbonero y otros, 2017; Mears y Marzal, 2018; Cardona, Unceta y Barandiarán, 2016; Contreras, 2018; Mastache y Devetac, 2017). 
A raíz de lo anterior, una visión más contemporánea de evaluación la define como un aspecto integral de la experiencia colaborativa de aprendizaje, por ende, los estudiantes aprenden mejor cuando sus experiencias universitarias exceden a la noción de recolección de actividades de los cursos, encontrándose diseñadas en un todo coherente (Biggs y Tang, 2011).

Hernández aborda la existencia de una relación entre la evaluación y el aprendizaje, que es generalmente problemática, y donde algunas de sus funciones son el reporte del logro de los estudiantes, la calificación y el apoyo en el proceso (2012: 490). Todo lo anterior, de acuerdo con la autora, representa la evaluación continua, que se identifica por combinar las funciones formativa y sumativa.

Existen estudios vinculados a las estrategias de evaluación coherentes con nociones más actuales (San Martín, Jiménez y Sánchez, 2016; Quesada, Rodríguez e Ibarra, 2017; Reyes, Ariza y Torreblanca, 2014; Rueda, Schmelkes y Díaz Barriga, 2014; Santos y Macedo, 2018). Una investigación desarrollada en un curso sobre evidencias concluyó que las evaluaciones formativas favorecen el logro de puntajes más altos en los exámenes finales. El estudio involucró a un grupo de control de 67 estudiantes y a un grupo de intervención de 51. Ambos grupos trabajaron con la estrategia de método de problemas y análisis de casos (Springer y Curcio, 2012).

Otro estudio se centró en identificar las percepciones de 10 académicos y 830 estudiantes de las carreras de Derecho y Psicología de una universidad holandesa en torno a la evaluación continua. A través de entrevistas semiestructuradas, se estableció que las percepciones hacia este tipo de evaluación eran positivas, pero el sentido varió en docentes y estudiantes. Mientras que en el primer grupo se destacó la ventaja de evaluar diversos tipos de conocimientos y habilidades, los estudiantes valoraron la posibilidad de poder testear anticipadamente los mismos conocimientos y habilidades requeridos en los exámenes finales (Day y otros, 2017).

Otra experiencia corresponde a la implementación del Aprendizaje Basado en Problemas en México, donde se identificó que, por medio de la promoción de una retroalimentación inmediata y la proporción de oportunidades para elaborar productos terminados, se alcanza una alta aceptación por parte de los estudiantes, especialmente en relación con la preparación para el mundo laboral, aspecto que fue recogido a partir de los resultados de la aplicación de una encuesta (Ramírez y Luna, 2017).

En Chile también se han realizado estudios en torno a las innovaciones asociadas a procesos de evaluación. Se implementó una experiencia de ensayo modificado utilizando encuestas dirigidas a docentes y estudiantes en conjunto con un grupo focal dirigido a estos últimos. Así, fue posible identificar ventajas y desafíos asociados a esta estrategia (González Marino, 2017).

Dentro de las ventajas, los participantes identificaron esta estrategia como una instancia que aporta a la construcción de aprendizajes. Por otra parte, consideran 
que permite desarrollar habilidades de resolución de problemas y que ofrece un acceso a retroalimentación oportuna. En tanto, dentro de los desafíos se identificó la necesidad de mejorar la estimación de tiempos asociados al proceso de respuesta de los estudiantes, lo que a su vez genera en ellos un sentimiento de ansiedad y presión. Complementariamente, se visualizaron las implicancias del diseño de los instrumentos de evaluación, dado que su construcción es más compleja que una evaluación tradicional (González Marino, 2017).

Las experiencias anteriores denotan un interés por innovar en la evaluación, aspecto que ha alcanzado un nivel de atención especial en la carrera de Derecho de la Universidad Católica de Temuco a partir del análisis del perfil de ingreso de sus estudiantes. Dentro de los diagnósticos y considerando competencias básicas para la carrera, se identifica que los estudiantes de la cohorte 2017 alcanzaron un $67 \%$ de aprobación en la prueba de comprensión lectora. Por otra parte, el promedio ponderado en la Prueba de Selección Universitaria de los mismos estudiantes fue de 546 puntos con una desviación estándar de un 52,5.

A raíz de las experiencias anteriores y las características del perfil de ingreso de los estudiantes de la cohorte 2017, se definió que en la carrera de Derecho algunas secciones de los cursos de Interpretación y Argumentación Jurídica e Introducción a la Resolución Colaborativa de Conflictos implementarían un piloto en sus planes de evaluación. Ambos cursos corresponden al segundo semestre del itinerario formativo de la carrera y los pilotos fueron aplicados por académicos vinculados a la Comunidad de Aprendizaje de Derecho de la Universidad Católica de Temuco, quienes participan constantemente en instancias de reflexión y formación docente, siguiendo las orientaciones del Modelo Educativo de la universidad. ${ }^{3}$

Es así como se implementó un plan de evaluación en cada curso compuesto por tres evaluaciones focalizadas ${ }^{4}$ aplicadas de manera previa a una evaluación integrada de desempeño..$^{5}$ Las primeras de ellas contaron con una ponderación menor a las evaluaciones integradas.

En estas evaluaciones se incorporaron estrategias como mapas conceptuales, realización de actividades a través de módulos e-learning, análisis de casos y un examen

3. Desde el año 2014, la carrera de Derecho ha implementado un plan renovado que recoge los cinco ejes del Modelo Educativo de la Universidad Católica de Temuco, saber: a) formación basada en competencias, b) aprendizaje significativo centrado en el estudiante, c) integración de tecnologías de la información y la comunicación, d) educación continua y e) formación humanista cristiana.

4. De acuerdo con las orientaciones de la Universidad Católica de Temuco, una evaluación focalizada permite que el estudiante demuestre la aplicación o utilización de contenidos (ya sean conceptuales, procedimentales o actitudinales) en determinadas situaciones o problemas profesionales.

5. Una evaluación integrada de desempeño está orientada a que el estudiante demuestre las actuaciones o desempeños profesionales en contexto, integrando y movilizando contenidos de diversa naturaleza y procedencia (también denominada evaluación compleja o auténtica). 
Tabla 1. Plan de Evaluación del curso Introducción a la Resolución Colaborativa de Conflictos

\begin{tabular}{|ll|}
\hline Tipo de evaluación & Ponderación \\
\hline 1. Evaluación prueba escrita-individual & $30 \%$ \\
\hline 2. Glosario de términos del curso-grupal & $10 \%$ \\
\hline 3. Mapa Conceptual-individual & $10 \%$ \\
4. Mapa Conceptual-grupal & $10 \%$ \\
\hline 5. Simulación caso negociación-grupal & $40 \%$ \\
6. Actividad e-learning & $0 \%$ \\
Nota de presentación de exámen $(1+2+3+4+5)$ & $70 \%$ \\
\hline Examen & $30 \%$ \\
\hline
\end{tabular}

Elaboración propia. Fuente: Universidad Católica de Temuco, 2017.

oral final. El propósito de esta variedad de evaluaciones era ofrecer oportunidades para evidenciar el avance de los estudiantes. Es importante precisar que tanto los mapas conceptuales, las actividades e-learning y los análisis de casos fueron evaluados con rúbricas y pautas de corrección que explicitan criterios asociados a los resultados de aprendizaje correspondientes. En la tabla 1 se presenta el plan de evaluación de uno de los cursos.

Un punto crítico en el diseño de la implementación del proyecto dice relación con el diseño de un examen final, que transformara el examen tradicional individual en una actividad integrada y basada en la utilización estrategias que permitan evaluar aprendizajes significativos.

De esta forma, se diseñó un examen en el que se entregaron, con el tiempo de antelación necesario, las pautas para la resolución de un caso o situación jurídica que involucra la aplicación de los contenidos del curso y que debía ser desarrollado a través de un trabajo grupal. Con esto, los estudiantes presentaron sus trabajos en forma oral ante sus compañeros y una comisión evaluadora.

Esta experiencia de innovación en los procedimientos de evaluación en derecho abre diversas interrogantes, por ejemplo, respecto a la percepción de los estudiantes hacia estos cambios. Aún cuando están fundamentados en buenas prácticas, se hace preciso generar estudios que permitan evaluar experiencias de innovación en la carrera de Derecho y en el marco de una formación por competencias.

Por otra parte, también será importante generar información que permita profundizar y mejorar los procesos de evaluación, aportar a una discusión necesaria sobre los procesos de formación de abogados y abrir espacios de reflexión respecto a los métodos de evaluación de los aprendizajes. 


\section{Metodología}

El presente estudio es descriptivo y de carácter mixto (Hernández, Fernández y Baptista, 2006), y está orientado a evaluar la implementación de un piloto de evaluación continua en cursos de primer año de la carrera de Derecho de la Universidad Católica de Temuco. En específico, se buscó identificar la percepción de los estudiantes de los cursos pilotos acerca de la evaluación en instancias previas y posteriores a la innovación. Por otra parte, se identificaron las relaciones entre las calificaciones obtenidas y los promedios finales de los cursos con evaluación continua.

\section{Participantes}

Los participantes fueron estudiantes de segundo semestre que asistieron a los cursos de Interpretación y Argumentación Jurídicas e Introducción a la Resolución Colaborativa de Conflictos. ${ }^{6}$ La distribución porcentual entre hombres y mujeres fue de $33,5 \%$ y $66,5 \%$, respectivamente.

\section{Instrumentos de recolección de información}

Los datos cuantitativos fueron obtenidos desde las actas de calificaciones de la carrera que participaron del piloto y de sus secciones paralelas (sin innovación).

Los datos cualitativos fueron recolectados por medio de dos cuestionarios (pre $y$ post) con preguntas abiertas enfocadas en identificar las percepciones de los estudiantes en torno a las estrategias de evaluación empleadas.

\section{Proceso de recolección de información}

El primer análisis se basó en la esquematización y observación de datos cuantitativos, los que corresponden a las calificaciones de 117 estudiantes. Para el análisis se consideraron a los estudiantes de 6 docentes diferentes, donde 4 desarrollaron cursos sin esta innovación y 2 implementaron la innovación presentada en este estudio.

En lo que respecta a los análisis cualitativos, el primer cuestionario se aplicó al inicio del semestre y con posterioridad a la primera semana de clases a través de la utilización del entorno virtual de aprendizaje institucional. El segundo cuestionario fue aplicado al finalizar el semestre y con anterioridad a la rendición del examen final del curso. A diferencia del cuestionario inicial, este fue aplicado en formato impreso al inicio de la clase. El cuestionario inicial contó con 45 participantes de tres secciones, mientras que el final contó con 95 participantes.

6. Del curso de Interpretación y Argumentación Jurídicas participaron dos secciones, mientras que en el curso de Introducción a la Resolución Colaborativa de Conflictos participó una sección. 


\section{Análisis de la información}

Los datos cuantitativos fueron procesados en el software SPSS versión 22. En primer lugar, se realizó una depuración de los datos por medio de la identificación y exclusión de datos atípicos univariantes y multivariantes a través de los puntajes $\mathrm{z}$ y la distancia de Mahalannobis (Myers, 2011).

Del proceso anterior se excluyeron dos casos del análisis. Posterior a esto, se llevó a cabo un análisis de estadística descriptiva basado en medidas de tendencia central y de dispersión. Para observar la asociación entre las variables, se realizó un análisis de tablas cruzadas y de correlación de Pearson, que considera únicamente a los docentes que implementaron la innovación, ya que no se tuvo acceso a todas las calificaciones de las demás secciones.

Para los cuestionarios, las respuestas fueron codificadas por medio del software Atlas ti, versión 8. La codificación fue realizada por medio de un análisis crítico del discurso (Gómez Cuevas, 2015). El esquema de codificación se basó en categorías diseñadas para capturar los temas dominantes presentes en las respuestas de los estudiantes.

\section{Resultados}

\section{Notas y promedios de cursos}

Al momento de analizar los datos de los cursos, es primordial establecer un análisis descriptivo basado en las medidas de dispersión y de tendencia central para establecer una primera visión sobre el comportamiento de los datos.

La tabla 2 muestra que existen dos grupos claramente distinguibles en cuanto a la nota del promedio final. La media nos denota que los profesores 1, 2, 3 y 4, quienes no implementaron la innovación, poseen una media entre un 4,3 a 5,3 en una escala de 1 a 7. La mediana aumenta en comparación con la media para los mismos docentes, la que establece que un 50\% de los estudiantes se encuentra entre una nota 1 y 5,4. Del mismo modo, la dispersión de las notas no posee mayor variabilidad, lo que significa que los promedios finales se encuentran cercanos a la media.

El siguiente grupo está compuesto por los profesores 5 y 6 , quienes implementaron evaluación continua. La media se posiciona entre el 5,9 y 6,1, respectivamente, en una escala de 1 a 7 . La mediana se establece en los mismos resultados de la media, lo que significa que existe una menor medida de dispersión de los datos y que puede existir una distribución normal. Una menor variabilidad se confirma con la desviación estándar, que es menor a 1.

Lo anterior expone la distribución de los datos asociados al promedio final del curso, no obstante, con la información presentada no se puede establecer que el método realizado por cada grupo de docentes sea más o menos efectivo. A diferencia de 
Tabla 2. Estadísticos descriptivos con respecto a la nota del promedio final

\begin{tabular}{|llcccccc|} 
& Método de evaluación & Media & Mediana & Moda & DS & Mínimo & Máximo \\
\hline Profesor 1 & Sin innovación & 4,383 & 4,600 & 4,5 & 1,356 & 1,0 & 6,0 \\
Profesor 2 & Sin innovación & 4,417 & 4,500 & 4,3 &, 779 & 1,0 & 5,7 \\
\hline Profesor 3 & Sin innovación & 4,831 & 5,000 & 5,1 & 1,3299 & 1,0 & 7,0 \\
Profesor 4 & Sin innovación & 5,305 & 5,400 & 5,3 & 1,1013 & 1,0 & 7,0 \\
Profesor 5 & Con innovación & 5,968 & 5,900 & 7,0 &, 7800 & 3,0 & 7,0 \\
\hline Profesor 6 & Con innovación & 6,100 & 6,100 & 7,0 &, 8213 & 1,6 & 7,0 \\
\hline
\end{tabular}

Elaboración propia. Fuente: Universidad Católica de Temuco, 2017.

Tabla 3. Relación entre la situación final del estudiante y la adscripción del docente

\begin{tabular}{|lccccc|}
\hline & Aprobado & Reprobado & $\begin{array}{c}\text { Reprobado por } \\
\text { inasistencia }\end{array}$ & $\begin{array}{c}\text { Porcentaje } \\
\text { total }\end{array}$ \\
\hline Docente con innovación & Sí & 98,3 & 0,9 & 0,9 & 100,0 \\
Docente sin innovación & No & 90,5 & 5,7 & 3,8 & 100,0 \\
\hline
\end{tabular}

Elaboración propia. Fuente: Universidad Católica de Temuco, 2017.

lo anterior, en la tabla 3 se observa que sí existe una relación entre la adscripción del docente y la tasa de aprobación de los estudiantes dependiendo de si se implementó la innovación o no.

En efecto, en los cursos implementados por los profesores 5 y 6 existe un mayor porcentaje de aprobación en comparación con los docentes sin la implementación de una evaluación continua.

La tabla 4 expone el nivel de asociación de las relaciones que se establecen entre los distintos tipos de evaluación y la nota del promedio final de los estudiantes de la carrera. Estos datos solo aplican a los profesores 5 y 6.

Se observa que existe una relación significativa entre el promedio final y la evaluación 1 , la que representa un 30\% del total de promedio de presentación a examen. Esta relación posee una fiabilidad buena de 0,554 (Cortés, Rubio y Gaitán, 2010), es decir, a medida que aumenta la nota de la evaluación 1 aumenta también la del promedio final. La evaluación 4 posee una relación moderada de o,448 con el promedio final del curso y posee una ponderación del 10\% del promedio de presentación. Finalmente, la evaluación 5 posee una correlación de 0,613 con el promedio final y representa un 40\% del promedio de presentación a examen.

\section{Cuestionario preliminar}

El primer cuestionario aplicado tuvo por objetivo identificar las percepciones de los estudiantes sobre las formas de evaluación experimentadas por ellos durante la carrera. La primera pregunta apuntaba a la descripción de las características de los medios 
Tabla 4. Asociación entre las evaluaciones y la nota del promedio final

\begin{tabular}{|c|c|c|c|c|c|c|c|c|c|}
\hline & & $\begin{array}{l}\text { Nota } \\
\text { Final }\end{array}$ & $\begin{array}{c}\text { Evaluación } \\
1(30 \%)\end{array}$ & $\begin{array}{c}\text { Evaluación } \\
2(10 \%)\end{array}$ & $\begin{array}{c}\text { Evaluación } \\
3(10 \%)\end{array}$ & $\begin{array}{c}\text { Evaluación } \\
4(10 \%)\end{array}$ & $\begin{array}{c}\text { Evaluación } \\
5(40 \%)\end{array}$ & Promedio & $\begin{array}{c}\text { Examen } \\
30 \%\end{array}$ \\
\hline \multirow{3}{*}{ Nota final } & $\begin{array}{l}\text { Correlación } \\
\text { Pearson }\end{array}$ & 1 &, $554^{* *}$ &, $269^{* *}$ &, $382^{* *}$ &, $448^{* *}$ &, $613^{* *}$ &, $782^{* *}$ &, $756^{* *}$ \\
\hline & $\begin{array}{l}\text { Sig. } \\
\text { (bilateral) }\end{array}$ & & ,000 &, 004 &, 000 &, 000 &, 000 & ,000 & ,000 \\
\hline & $\begin{array}{l}\text { Correlación de } \\
\text { Pearson }\end{array}$ & & 1 & 139 & ,139 &, $395^{* *}$ &, $262^{* *}$ &, $724^{* *}$ &, $389^{* *}$ \\
\hline \multirow[t]{2}{*}{$\begin{array}{l}\text { Evaluación } \\
1(30 \%)\end{array}$} & Sig. (bilateral) & & & 140 & ,138 & ,000 &, 005 &, 000 & ,000 \\
\hline & $\begin{array}{l}\text { Correlación de } \\
\text { Pearson }\end{array}$ & & & 1 & ,028 & $260^{* *}$ &, $233^{*}$ &, $331^{* *}$ &, $185^{*}$ \\
\hline \multirow{2}{*}{$\begin{array}{l}\text { Evaluación } \\
2(10 \%)\end{array}$} & Sig. (bilateral) & & & & 770 & ,005 & ,012 & ,000 & ,047 \\
\hline & $\begin{array}{l}\text { Correlación de } \\
\text { Pearson }\end{array}$ & & & & 1 & , 134 &, $298^{* *}$ &, $367^{* *}$ &, $296^{* *}$ \\
\hline \multirow[t]{2}{*}{$\begin{array}{l}\text { Evaluación } \\
3(10 \%)\end{array}$} & Sig. (bilateral) & & & & & 153 & ,001 &, 000 &, 001 \\
\hline & $\begin{array}{l}\text { Correlación de } \\
\text { Pearson }\end{array}$ & & & & & 1 &, $278^{* *}$ &, $603^{* *}$ &, $452^{* *}$ \\
\hline \multirow[t]{2}{*}{$\begin{array}{l}\text { Evaluación } \\
4(10 \%)\end{array}$} & Sig. (bilateral) & & & & & & ,003 &, 000 & ,000 \\
\hline & $\begin{array}{l}\text { Correlación de } \\
\text { Pearson }\end{array}$ & & & & & & 1 &, $792^{* *}$ &, $347^{* *}$ \\
\hline \multirow[t]{2}{*}{$\begin{array}{l}\text { Evaluación } \\
5(40 \%)\end{array}$} & Sig. (bilateral) & & & & & & & ,000 &, 000 \\
\hline & $\begin{array}{l}\text { Correlación de } \\
\text { Pearson }\end{array}$ & & & & & & & 1 &, $547^{* *}$ \\
\hline Promedio & Sig. (bilateral) & & & & & & & & ,000 \\
\hline
\end{tabular}

Elaboración propia. Fuente: Universidad Católica de Temuco, 2017.

de evaluación. En respuesta, los estudiantes exponen que ayudan a la comprensión de los contenidos por medio del raciocinio, lo que favorece la comprensión y la relación entre los contenidos.

En efecto, los estudiantes poseen una valoración positiva frente a las nuevas formas de evaluación y afirman que «la forma de evaluar es bastante más eficiente que el sistema antiguo, que requiere solo memorización sin que propicie un verdadero aprendizaje. Es el camino a seguir. Por eso debe masificarse esta forma de evaluación» (Participante 3, 2017). Las opiniones positivas se centran en una percepción sobre el tipo de aprendizaje, lo que posiciona como negativa a la memorización sin una relación entre los contenidos. Para complementar lo anterior, se expone que «es muy necesario que al estudiar cierta materia el alumno posea un conocimiento acerca de todo, más comprensión y no memorización, por lo cual las pruebas orales no son un buen método para evaluar» (Participante 2, 2017). 
Las opiniones negativas fueron poco frecuentes. En relación con esto, algunos estudiantes señalaron que este cambio no es el adecuado, ya que existe un método de evaluación establecido y validado que se acopla con el perfil de egreso del abogado. Para complementar lo anterior, un estudiante afirma que «ya tenemos evaluaciones en las cuales tenemos un aprendizaje adecuado y reemplazarlo por más evaluaciones no me parece correcto» (Participante 1, 2017).

A esto se agrega la opinión del participante 18, quien expresa lo siguiente: «No me parece e incluso sacaría de la malla la realización de tantos trabajos, los que quitan tiempo para aprender lo teórico, un aspecto que para un abogado es sumamente importante». En efecto, los medios de evaluación desde la percepción de este estudiante deben vincularse al ejercicio profesional, ya que afirma lo siguiente: «No creo que sea necesario un cambio en la formación evaluativa de la carrera, necesitamos pruebas orales y escritas como una forma de fortalecer nuestro carácter como abogados».

Estos estudiantes consideran que la evaluación no debiera modificarse en función de lo que es esperable dentro de la formación de abogados, es decir, con un foco en el aprendizaje de contenidos de la asignatura.

\section{Cuestionario final}

Los resultados del cuestionario final fueron obtenidos con posterioridad a la implementación de la innovación. La primera pregunta se centró en identificar la percepción sobre las ventajas o desventajas de esta experiencia desde la perspectiva de los estudiantes.

Casi la totalidad de los estudiantes considera que es positivo participar de este tipo de innovaciones, ya que trae consigo ventajas para su proceso formativo. La utilización de distintos métodos permite identificar habilidades y deficiencias. Además, consideran que la integración de los contenidos con ejemplos reales en su aplicación no solo ayuda al aprendizaje, sino también al fortalecimiento del trabajo en equipo. Por otra parte, destacan la responsabilidad de los compañeros. A raíz de lo anterior, un estudiante expresa que "favorece el trabajo en equipo, nos permite complementar experiencias y conocimientos e incorpora nuevas tecnologías para nuestro aprendizaje como estudiantes de Derecho» (Participante 4, 2017).

Existe un número marginal de estudiantes que opinan que este nuevo método de innovación es desfavorable, ya que afirman estar acostumbrados a las clases presenciales, por lo tanto, cambiar a una modalidad e-learning complejiza el proceso para ellos, ya que como abogados deben interactuar con la gente de manera personal, no de forma online (Participante 9, 2017).

En un segundo aspecto, se consultó sobre las ventajas y desventajas en relación con el acceso y al uso de los módulos e-learning. En general, los estudiantes consideran que el acceso es amplio y el uso de la plataforma es simple. La herramienta $e$ - 
learning proporciona facilidades al momento de estudiar y es más versátil e integral, lo que ayuda a administrar el tiempo de mejor forma como también llevar un orden de los contenidos y las evaluaciones. Sobre este punto se afirma que «la herramienta e-learning proporciona facilidades de estudio mucho más versátiles y, por lo tanto, más integrales en cuanto a la administración de tiempos personales para el estudio» (Participante 3, 2017).

Lo anterior se refleja en el tipo de aprendizaje alcanzado por este estudiante, quien expresa que le parece favorable «debido a la oportunidad de orientar y guiar el autoaprendizaje dirigido y no impuesto de formas tradicionales ortodoxas, además de ineficientes en tiempos actuales» (Participante 9, 2017). El trabajo autónomo y dirigido permite una retroalimentación constante por parte de los estudiantes que dé cuenta de los propios errores y, así mismo, una corrección de estos en el transcurso de la asignatura.

Finalmente, algunos estudiantes señalaron que no existió un aprendizaje profundo y que prefieren el método tradicional de evaluación, ya que consideran que las clases presenciales contribuyen de mejor forma al aprendizaje de los estudiantes. Lo anterior se fundamenta en la siguiente opinión: «Todavía prefiero el método tradicional, ya que es más fácil acceder al profesor» (Participante 17, 2017).

\section{Conclusión}

Este estudio ha permitido identificar, por una parte, que existe una relación entre la evaluación continua y los promedios finales de los estudiantes. Esto se sustenta en la comparación con los resultados obtenidos en secciones paralelas, en las que no se aplicó la innovación en el plan de evaluación. Estos resultados complementan lo identificado en el estudio de Springer y Curcio (2012) en relación con el efecto de la evaluación continua en los exámenes finales de un curso.

La enseñanza tradicional del Derecho ha estado centrada en la evaluación de los resultados y ha dejado en un segundo plano la evaluación del proceso. En este marco, los exámenes finales se transforman en una instancia decisiva para el estudiante. Así, la evaluación continua permitió realizar un seguimiento de los avances de los estudiantes, combinando, tal como lo indica Hernández (2012), la función formativa a través de la retroalimentación y la función sumativa como aporte a la validación de los aprendizajes.

En segundo lugar, en relación con el análisis cuantitativo, se pudo establecer para este caso que las tasas de aprobación de los cursos con innovación fueron ligeramente más altas que en las que este tipo de innovación no estuvo presente.

Por último, se pudieron establecer correlaciones entre los resultados obtenidos por los estudiantes en algunas evaluaciones y el promedio final del curso. Las evaluaciones 1,4 y 5 corresponden a una prueba con preguntas orientadas al análisis 
de casos, el desarrollo de un mapa conceptual grupal y una simulación, respectivamente. En ellas, los estudiantes debían demostrar el logro de habilidades de alto orden, mientras que en las evaluaciones 2 y 3 , correspondientes a la elaboración de un glosario y un mapa conceptual individual, apuntaban a habilidades de bajo orden. Lo anterior podría implicar la existencia de una mayor fiabilidad de las primeras en comparación con las segundas.

En relación con lo anterior, se sostiene lo indicado por Donald (2009) respecto a la existencia de un vínculo directo de la enseñanza del derecho con el desarrollo de habilidades de alto orden.

Es importante destacar que la evaluación correspondiente al desarrollo de un mapa conceptual grupal con $10 \%$ de ponderación alcanzó un coeficiente de 0,448 . Por otra parte, la evaluación de la simulación alcanzó un coeficiente de 0,782 con una ponderación del 40\%. Como fue mencionado anteriormente, ambas comparten la característica de ser evaluaciones integradas de desempeño que apuntan a la evaluación de habilidades de alto orden. Por tanto, si bien no es posible afirmar la existencia de una causalidad, la relación entre estas variables con el promedio final no es descartable como factor explicativo.

Por otra parte, desde el análisis cualitativo de los cuestionarios se identificó que las percepciones de algunos estudiantes aún reflejan una preferencia hacia una evaluación tradicional. Desde la perspectiva de este grupo de estudiantes, existe una visión sobre el perfil de egreso que condiciona lo que se percibe como adecuado o inadecuado en relación con la evaluación. Lo anterior refleja que las visiones tradicionales de la enseñanza del Derecho no solo están presentes en las creencias docentes, sino también en las que pertenecen a los estudiantes. Esto coincide con el concepto de pedagogías características, ya que la formación del Derecho se asocia a personalidades, disposiciones y culturas propias (Shulman, 2005).

Por otra parte, fue posible identificar a estudiantes que valoraron los ajustes realizados y que destacaron su aporte en la construcción de nuevos aprendizajes disciplinares que exceden a la memorización de contenidos. Un ejemplo de lo anterior es el caso de los análisis de casos, donde se invitó a los estudiantes a aplicar ciertos procedimientos en la resolución de conflictos. Esto se sustenta bajo la mirada de Brown (2011: 92), quien indica que el contenido debe ser considerado un medio y no un fin en sí mismo.

Los estudiantes destacan también que esta innovación favorece el desarrollo de competencias genéricas necesarias en la formación, como el aprendizaje autónomo, la integración de tecnologías y el trabajo colaborativo. Este aspecto también fue observado en el estudio de González, donde la estrategia implementada permitió el desarrollo de habilidades de resolución de problemas (González Marino, 2017).

Un último aspecto destacado en el análisis cualitativo corresponde a la retroalimentación. El diseño de un plan de evaluación que incorpore evaluaciones integra- 
das de desempeño y focalizadas, con criterios y estándares claros y definidos, permite lograr una retroalimentación más efectiva. Esto fue valorado por los estudiantes, ya que les permitió corregir sus errores a tiempo y aplicar remediales de manera oportuna. Esto se relaciona con la incidencia que la evaluación tiene en el proceso de formación (Biggs y Tang, 2011).

Las próximas líneas de investigación se desarrollarán en función de generar una caracterización del perfil del estudiante de la carrera de Derecho de la Universidad Católica de Temuco. El objetivo será conocer, con mayor profundidad, las expectativas en relación con su formación y cómo esto se vincula con el quehacer profesional del egresado.

En cuanto a una segunda línea de investigación, se propone indagar de forma comparativa en cursos con métodos innovadores de evaluación y métodos tradicionales de evaluación.

\section{Referencias}

BENFELD, Johann (2017). «El nuevo paradigma universitario de acceso universal: Su origen, características y alcances en relación a la enseñanza del derecho». Revista Chilena de Derecho, 44 (2): 575-597. Disponible en bit.ly/2E4LVgB.

BIGgS, John y Catherine TANG (2011). Teaching for Quality Learning at University. Nueva York: Open University Press.

Brown, Gloria (2011). «Student-centered learning in higher education». International Journal of Teaching and Learning in Higher Education, 23 (3): 92-97. Disponible en bit.ly/2zLISI4.

Brown, Sally (2015). «Perspectivas internacionales sobre la práctica de la evaluación en educación superior». Relieve. Revista Electrónica de Investigación y Evaluación Educativa, 21 (1): 1-15. Disponible en bit.ly/2rlZMHz.

Carbonero, Miguel Ángel, Luis Martín Antón, Valle Flores y Alvaci Freitas Resende (2017). «Estudio comparado de los estilos de enseñanza del profesorado universitario de ciencias sociales de España y Brasil». Revista Complutense de Educación, 28 (2): 631-647. Disponible en bit.ly/2UiXEOc.

Cardona Rodríguez, Antonio, Koldo Unceta Satrustegui y Marta BarandiaRÁN GALDós (2016). «La pertinencia integral en la evaluación de la calidad de la educación superior». Cultura y Educación: Culture and Education, 28 (2): 351-358. Disponible en bit.ly/2zJWsdP.

Coloma Correa, Rodrigo (2005). «El ocaso del profesor Binns: Un ensayo acerca de la enseñanza del derecho en Chile». Ius et Praxis, 11 (1): 133-172. DOI: 10.4067/ So718-00122005000100006.

Contreras, Gloria (2018). «Retroalimentación por pares en la docencia universi- 
taria. Una alternativa de evaluación formativa». Formación Universitaria, 11 (4): 83-94. Disponible https://bit.ly/2zLu2Aj.

Cortés, Edgar, Jorge Rubio y Hernando Gaitán (2010). «Métodos estadísticos de evaluación de la concordancia y la reproducibilidad de pruebas diagnósticas». Revista Colombiana de Obstetricia y Ginecología, 61 (3): 247-255. Disponible en bit. ly/2h7aiki.

Day, Indira, Floris Van Blankenstein, Michiel Westenderg y Wildried Admiraal (2017). «Teacher and student perceptions of intermediate assessment in higher education». Educational Studies, 44 (4): 449-467. DOI: 10.108o/03055698.2017.1382324.

De Miguel, Mario y Pedro Apodaca (2009). «Criterios para evaluar el impacto de los Planes de Evaluación de la Calidad de la Educación Superior. Criteria for evaluating the impact of Higher Education Quality Assessment Plans». Revista de Educación, 349: 295-310.

Donald, Janet (2009). «The Commons: Disciplinary and Interdisciplinary encounters». En The University and Its Disciplines: Teaching and Learning within and Beyond Disciplinary Boundaries. Nueva York: Routledge.

FInK, Dee (2003). Creating Significant Learning Experiences: An Integrated Approach to Designing College Courses. San Francisco: Jossey-Bass.

Fry, Heather, Steve Ketteridge y Stephanie Marshall (2015). A Handbook for Teaching in Higher Education: Enhancing Academic Practice. Nueva York: Routledge.

GABILONDO, José (2017). «El método de casos en la pedagogía jurídica de los Estados Unidos: Una valoración subjetiva». Revista Pedagogía Universitaria y Didáctica del Derecho, 2 (4): 74-114. DOI: 10.5354/0719-5885.2017.46251.

Gómez Cuevas, Héctor (2015). "Análisis crítico del discurso al campo del currículum de la formación inicial docente en Chile». Estudios Pedagógicos, 41 (1): 311322. Disponible en bit.ly/2EgKOoz.

Gómez Francisco, Taeli y Juan Rubio González (2017). «Cognición contextualizada: Una propuesta didáctica y psicopedagógica socioconstructivista para la enseñanza-aprendizaje del derecho». Revista Pedagogía Universitaria y Didáctica del Derecho, 4 (2): 40-63. DOI: 10.5354/0719-5885.2017.47970.

GonZÁlez MARINo, Israel (2017). «Adaptación e implementación del ensayo modificado en la didáctica del derecho». Revista Pedagogía Universitaria y Didáctica del Derecho 4 (1): 115-145. DOI: 10.5354/0719-5885.2017.6252.

HALPERN F., Diane (2007). «The Nature and Nurture of Critical Thinking». En Robert J. Sternberg, Henry L. Roediger y Diane F. Halpern (editores), Critical Thinking in Psychology. Nueva York: Cambridge University Press.

Hernández, Rebeca, Roberto Fernández y Pilar Baptista (2006). Metodología de la Investigación. México: McGraw-Hill Interamericana.

Hernández, Rosario (2012). «Does continuous assessment in higher education support student learning? Higher Education, 64. DOI: 10.1007/s10734-012-9506-7. 
Hunt, Lynne y Denise Chalmers (2013). University Teaching in Focus: A learningcentred approach. Nueva York: Routledge.

Mastache, Anahí y Roald Devetac (2017). «La formación jurídica: una mirada desde una didáctica analítica y reflexiva». Academia: Revista sobre Enseñanza del Derecho de Buenos Aires, 15 (30): 153-18o. Disponible en bit.ly/2Ed6k3X.

Mears Delgado, Berenice y Miguel Ángel Marzal (2018). «Evalfin: Un modelo de evaluación de alfabetización informacional para instituciones de educación superior». El Profesional de la Información, 27 (4): 879-89o. Disponible en bit. ly/2DZV89E.

Myers, Teresa (2011). «Goodbye, Listwise Deletion: Presenting Hot Deck Imputation as an Easy and Effective Tool for Handling Missing Data». Communication Methods and Measures, 5 (4):297-310. Disponible en bit.ly/2EhnNYZ.

NuCKLEs, Charles (2000). «Student-Centered Teaching: Making It Work». Adult Learning, 11: 5-6. Disponible en bit.ly/2KY 4vbF.

Padilla Carmona, María Teresa y Javier Gil Flores (2008). «La evaluación orientada al aprendizaje en la Educación Superior: condiciones y estrategias para su aplicación en la docencia universitaria». Revista Española de Pedagogía, 66 (241): 467-485. Disponible en bit.ly/2StDm2I.

PÉrez Villar, Carmen (2015). «Enseñar derecho de forma colaborativa. Ensayo sobre una primera experiencia». Revista Pedagogía Universitaria y Didáctica del Derecho 1 (1): 88-103. Disponible en https://bit.ly/2PlY2bo.

Quesada Serra, Victoria, Gregorio Rodríguez y María Soledad Ibarra (2017). «Planificación e innovación de la evaluación en educación superior: la perspectiva del profesorado». Revista de Investigación Educativa, 35 (1): 53-70. Disponible en bit.ly/2UiKyjJ.

Ramírez Mejía, Samuel (2016). «La aplicación de exámenes en forma colaborativa: Una forma de reforzar la función pedagógica en la evaluación del aprendizaje del derecho». Ponencia del Congreso CEEAD en Monterrey, Nuevo León, México.

RAmírez, Patricia e Ignacio Luna (2017). «Aprendizaje basado en problemas: Metodología didáctica para abordar la asignatura de Derecho Procesal Civil». Revista Pedagogía Universitaria y Didáctica del Derecho 1 (4): 189-224. Disponible en bit. ly/2RCkdf5.

Reyes Carreto, Ramón, Francisco Ariza y Omar Torreblanca (2014). «Un modelo empírico para explicar el desempeño académico de estudiantes de bachillerato». Perfiles educativos, 36 (146): 45-62. Disponible en bit.ly/2BTbYFS.

Rueda Beltrán, Mario, Sylvia Schmelkes y Ángel Díaz BARriga (2014). «La evaluación educativa. Presentación del número especial de Perfiles Educativos 2013. La evaluación en la educación superior». Perfiles educativos, 36 (145): 190-204. Disponible en bit.ly/2BTk812. 
SANtos, Jackson y Fernanda Macedo (2018). «Reflexões sobre o educador jurista e a concretude da educação jurídica no ensino superior». Revista de Pesquisa e Educação Jurídica, 4 (1): 95-111. Disponible en bit.ly/2PiYIxD.

San Martín, Sonia, Nadia Jiménez y Estefanía SÁnchez (2016). «La evaluación del alumnado universitario en el Espacio Europeo de Educación Superior». Aula Abierta, 44 (1): 7-14. Disponible en bit.ly/2rls2tN.

Shulman, Lee S. (2005). «Signature pedagogies in the professions». Daedalus 134 (3): 52-59. Disponible en bit.ly/2zGJuh6

Springer Sargent, Carol y Andrea A. Curcio (2012). «Empirical Evidence That Formative Assessments Improve Final Exams». Journal of Legal Education 61 (3): 379-405. Disponible en bit.ly/2BSQDfW.

WeIMer, Maryellen (2013). Learner-centered teaching: Five Key Changes to Practice. San Francisco: Jossey-Bass.

Wiggins, Grant y Jay McTighe (2005). Understanding by design. Alexandria: ASCD.

\section{Sobre los autores}

Alejandra Cid Droppelmann es académica de planta, jefa de la carrera de Derecho de la Universidad Católica de Temuco, licenciada en Ciencias Jurídicas por la Universidad Católica de Temuco, magíster en Derecho Penal y Nuevo Proceso Penal por la Universidad Mayor y diplomada en Metodología de la Enseñanza del Derecho por la Universidad Católica de Temuco.Su correo electrónico es acid@uct.cl.

Beatriz Moya Figueroa es Asesora Pedagógica en el Centro de Desarrollo e Innovación de la Docencia de la Universidad Católica de Temuco, licenciada en Educación, profesora de Inglés, Master of English as a Foreign Language por la Universidad Católica de Temuco y diplomada en Gestión y Evaluación de Proyectos en Educación Superior por la Pontificia Universidad Católica de Chile. Su correo electrónico es bmoya@uct.cl.

Patricia Toledo Zúñiga es académica de planta permanente del Instituto de Derecho Público de la Universidad Austral de Chile, licenciada en Ciencias Jurídicas por la Universidad Católica de Temuco, abogada, doctora en Derecho por la Universidad Pompeu Fabra de Barcelona y Master Avanzado en Ciencias Jurídicas por la misma casa de estudios. Su correo electrónico es: patricia.toledo@uach.cl.

Felipe Quintano Méndez es sociólogo, profesional de apoyo en el Centro de Desarrollo e Innovación de la Docencia de la Universidad Católica de Temuco, diplomado en Estadística Aplicada por la Universidad Austral de Chile y estudiante de Magíster en Psicología en la Universidad de la Frontera. Su correo electrónico es fquintano@uct.cl. 
La Revista Pedagogía Universitaria y Didáctica del Derecho (RPUDD) es una publicación científica semestral que contribuye a la reflexión multidisciplinaria sobre pedagogía universitaria y didáctica del derecho, para la formación y consolidación de esta área de investigación; así como a la difusión de prácticas innovadoras en la enseñanza-aprendizaje del derecho considerando el contexto nacional e internacional. Es una publicación electrónica internacional con una codirección entre Brasil y Chile.

\author{
DIRECTORA \\ María Francisca Elgueta Rozas \\ Universidad de Chile \\ DIRECTOR \\ Renato Duro Dias \\ Universidad Federal de Rio Grande, Brasil \\ SITIO WEB \\ pedagogiaderecho.uchile.cl \\ CORREO ELECTRÓNICO \\ rpedagogia@derecho.uchile.cl \\ LICENCIA DE ESTE ARTÍCULO
}

Creative Commons Atribución Compartir Igual 4.o Internacional

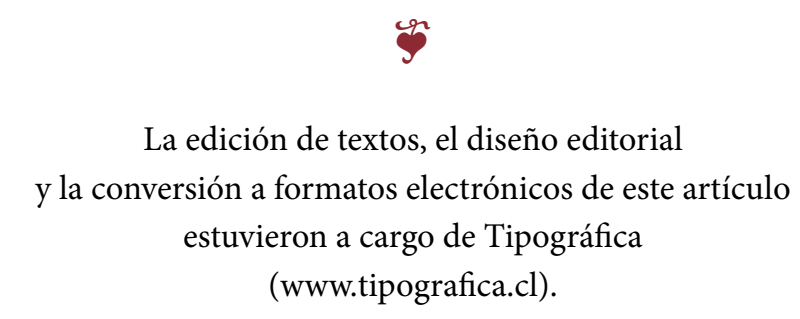

